\title{
Asymptotic Wave Functions and Energy Distributions for Symmetric Hyperbolic Systems of First Order
}

By

Kiyosi Krtahara*

\section{§1. Introduction}

This paper studies from the view point of $L^{2}$-theory the asymptotic behavior for $t \rightarrow \infty$ of solutions (with finite energy) of symmetric hyperbolic systems of first order with constant coefficients. For each solution of such systems the corresponding asymptotic wave function will be constructed from the initial data. The asymptotic energy distributions of the solutions will be investigated making use of the asymptotic wave functions.

Wilcox [8] studied these problems for solutions of the wave equation

$$
\frac{\partial^{2} v}{\partial t^{2}}-\frac{\partial^{2} v}{\partial x_{1}^{2}}-\cdots-\frac{\partial^{2} v}{\partial x_{n}^{2}}=0
$$

where $t \in \boldsymbol{R}, x=\left(x_{1}, \ldots, x_{n}\right) \in \boldsymbol{R}^{n}$ and $v=v(t, x)$ is a real valued function. He constructed asymptotic wave functions and calculated using these functions the asymptotic energy distributions of solutions for $t \rightarrow \infty$ in subsets of $\boldsymbol{R}^{n}$. Further he generalized these results to solutions of the wave equation in exterior domains [9].

The purpose of this paper is to extend his results in $\mathbb{R}^{n}$ to symmetric hyperbolic systems of first order with constant coefficients which satisfy the conditions stated below.

Consider the first order symmetric hyperbolic system

Communicated by S. Matsuura, October 8, 1975.

* Institute of Mathematics, The University of Tsukuba, Ibaraki 300-31, Japan. 


$$
L[V(t, x)]=\left(I \frac{\partial}{\partial t}+\sum_{j=1}^{n} A_{j} \frac{\partial}{\partial x_{j}}\right) V(t, x)=0
$$

with the initial condition

$$
V(0, x)=h(x),
$$

where $I$ is the unit matrix of order $N$, the $A_{j}$ are $N \times N$ constant hermitian matrices and $V(t, x)$ and $h(x)$ are vector-valued function whose values lie in $N$-dimensional complex space $C^{N}$. Let $P(\lambda, \xi)$ be the characteristic polynomial associated with the operator $L$;

$$
P(\lambda, \xi)=\operatorname{det}\left(\lambda I-\sum_{j=1}^{n} \xi_{j} A_{j}\right)
$$

where $\xi$ and $\lambda$ denote the dual variables of $x$ and $t$ respectively. The polynomial $P$ has the factorization

$$
P(\lambda, \xi)=P_{0}(\lambda, \xi)^{\beta_{0}} P_{1}(\lambda, \xi)^{\beta_{1}} \cdots P_{M}(\lambda, \xi)^{\beta_{M}}
$$

where the factors $P_{j}(\lambda, \xi)$ are distinct homogeneous polynomials in $(\lambda, \xi)$ and irreducible in the polynomial ring $C\left[\lambda, \xi_{1}, \ldots, \xi_{n}\right]$ of $(n+1)$ variables over the complex number field $\mathbb{C}$. Since the coefficients of $\lambda^{N}$ in $P(\lambda, \xi)$ is 1 , the factors are unique, apart from their order, by requiring the coefficients of the highest power of $\lambda$ in each $P_{j}(\lambda, \xi)$ be 1 .

We recall that a homogeneous polynomial $Q(\lambda, \xi)$ is said to be strictly hyperbolic (with respect to the vector $(1,0, \ldots, 0)$ ) if for every real $\xi \in \boldsymbol{R}^{n} \backslash\{0\}$ the roots of the equation $Q(\lambda, \xi)=0$ in $\lambda$ are real and distinct. Let $Q(\lambda, \xi)$ be a homogeneous hyperbolic polynomial of order $\mu$ and assume that $Q(0, \xi) \neq 0$ for any $\xi \in \boldsymbol{R}^{n} \backslash\{0\}$. Then $\mu$ is even and the roots of $Q(\lambda, \xi)=0$ can be enumerated so that

$$
\lambda_{m}(\xi)>\cdots>\lambda_{1}(\xi)>0>\lambda_{-1}(\xi)>\cdots>\lambda_{-m}(\xi), \quad \mu=2 m .
$$

It is easy to verify that the $\lambda_{k}(\xi)$ are real analytic functions of $\xi$ in $\boldsymbol{R}^{n} \backslash\{0\}$ and positively homogeneous of degree 1 . Further we have

$$
\lambda_{-k}(\xi)=-\lambda_{k}(-\xi), \quad|k|=1,2, \ldots, m .
$$

Now we state the conditions that we impose on the operator $L$. 
(L.1) In the factorization (1.4) of $P(\lambda, \xi), P_{0}(\lambda, \xi) \equiv \lambda$ and the $P_{j}(\lambda, \xi)$, $j=1, \ldots, m$ are strictly hyperbolic polynomials such that $P_{j}(0, \xi) \neq 0$ for any $\xi \in \boldsymbol{R}^{n} \backslash\{0\}$;

$$
P_{j}(\lambda, \xi)=\prod_{|k|=1}^{m_{j}}\left(\lambda-\lambda_{j, k}(\xi)\right), \quad j=1, \ldots, M
$$

where

$$
\lambda_{j, m_{j}}(\xi)>\cdots>\lambda_{j_{i} 1}(\xi)>0>\lambda_{j,-1}(\xi)>\cdots>\lambda_{j,-m_{j}}(\xi)
$$

From (L.1) it follows that the normal or slowness surface $S=\{s$; $P(1, s)=0\}$ of the operator $L$ consists of $\sum_{j=1}^{M} m_{j}$ sheets $S_{j, k}=\left\{s ; \lambda_{j, k}(s)=1\right\}$, $j=1, \ldots, M, k=1, \ldots, m_{j}$ which are analytic closed hypersurfaces enclosing the origin in $\boldsymbol{R}^{n}$. Then we assume

(L.2) The Gaussian curvature $K_{j, k}(s)$ of $S_{j, k}$ never vanishes anywhere in $S_{j, k}\left(j=1, \ldots, M, k=1, \ldots, m_{j}\right)$.

Remark. The condition (L.1) can be replaced by the following weaker one. In fact one can discuss by Appendix Theorem 4.1 in the same way as under the condition (L.1).

(L.1)' The factorization into linear factors in $\lambda$ of $P(\lambda, \xi)$ admits the form

$$
P(\lambda, \xi)=\lambda^{\alpha_{0}} \prod_{j=1}^{l}\left(\lambda-\lambda_{j}(\xi)\right)^{\alpha_{j}}
$$

where the $\alpha_{j}(0 \leqq j \leqq l)$ are constants and the $\lambda_{j}(\xi)(1 \leqq j \leqq l)$ are real valued analytic functions of $\xi$ in $\boldsymbol{R}^{n} \backslash\{0\}$ which satisfy

$$
\lambda_{j}(\xi) \neq 0 \text { for } 1 \leqq j \leqq l \text { and } \lambda_{j}(\xi) \not \equiv \lambda_{k}(\xi) \text { for } j \neq k, 1 \leqq j, k \leqq l
$$

To state our main theorem, we introduce the following notation. We denote by $\Theta$ the unit sphere in $\boldsymbol{R}^{n}$ and by $s_{j, k}(\theta)$ the inverse of Gauss $\operatorname{map}^{\dagger}: S_{j, k} \rightarrow \Theta$ for each $(j, k)$ with $1 \leqq j \leqq M$ and $1 \leqq k \leqq m_{j}$. We define

\footnotetext{
${ }^{\dagger}$ For the definition see for example Sternberg [6] or Matsumura [4].
} 


$$
\lambda_{j, k}^{*}(x)=|x| /\left|\operatorname{grad} \lambda_{j, k}\left(s_{j, k}(\theta)\right)\right|, \quad x=|x| \theta \in \boldsymbol{R}^{n} \mid\{0\} .
$$

$L^{2}\left(\boldsymbol{R}^{n}\right)$ denotes the Hilbert space of complex valued square integrable functions $v$ in $\boldsymbol{R}^{n}$ with norm

$$
\|v\|=\left\{\int_{R^{n}}|v(x)|^{2} d x\right\}^{\frac{1}{2}} .
$$

A $\boldsymbol{C}^{N}$-valued function $V \in L^{2}\left(\boldsymbol{R}^{n}\right)$ means that all the components of $V$ belong to $L^{2}\left(\boldsymbol{R}^{n}\right)$.

Under the conditions (L.1) and (L.2), we have

Theorem 1.1. For every solution $V(t, \cdot) \in L^{2}\left(\boldsymbol{R}_{x}^{n}\right)(t \geqq 0)$ of the equation (1.1), there exists an asymptotic wave function $V^{\infty}(t, \cdot) \in L^{2}\left(\boldsymbol{R}^{n}\right)$ $(t \geqq 0)$ such that

$$
\lim _{t \rightarrow \infty}\left\|V(t, \cdot)-V_{0}(\cdot)-V^{\infty}(t, \cdot)\right\|=0,
$$

where $V_{0}(x)$ is the static solution corresponding to the initial value $V(0, x)$ (if exists) and $V^{\infty}(t, x)$ has the form

$$
V^{\infty}(t, x)=\sum_{j=1}^{M} \sum_{|k|=1}^{m_{j}} V_{j, k}^{\infty}(t, x)
$$

and

$$
\begin{gathered}
V_{j, k}^{\infty}(t, x)=G_{j, k}\left(\lambda_{j,|k|}^{*}(x)-t, \theta\right) \sqrt{\lambda_{j,|k|}^{*}(\theta)}|x|^{-(n-1) / 2}, \\
x=|x| \theta \in \boldsymbol{R}^{n},
\end{gathered}
$$

where $G_{j, k}(r, \theta) \in L^{2}(\boldsymbol{R} \times \Theta)$ will be defined in terms of the initial value $V(0, x)$ and called asymptotic wave profile. ${ }^{\dagger}$ If the $A_{j}$ are real symmetric and the initial data $V(0, x)$ is a real valued function, $V^{\infty}(t, x)$ is also real valued.

This theorem is used to calculate the asymptotic distribution of the energy of $V(t, x)$ in bounded and unbounded subsets of $\boldsymbol{R}^{n}$. In fact, we have the following corollaries.

† As to the explicit formula, see (3.11) and (3.12). 
Corollary 1.2. For every bounded measurable subset $K$ of $\boldsymbol{R}^{n}$ we have

$$
\lim _{t \rightarrow \infty} \mathscr{E}(V, K, t)=\mathscr{E}\left(V_{0}, K, 0\right),
$$

where $\mathscr{E}(V, K, t)$ denotes the energy of $V(t, x)$ on $K$ :

$$
\mathscr{E}(V, K, t)=\int_{K}|V(t, x)|^{2} d x
$$

and if the static solution does not exist, we define $\mathscr{E}\left(V_{0}, K, 0\right)=0$.

Corollary 1.3. For any measurable cone $C \subset \boldsymbol{R}^{n}$ with its vertex at the origin, the asymptotic energy distribution

$$
\mathscr{E}^{\infty}(V, C) \equiv \lim _{t \rightarrow \infty} \mathscr{E}(V, C, t)
$$

exists.

Corollary 1.4. For any measurable cone $C \subset \boldsymbol{R}^{n}$ with its vertex at the origin and for any point $\bar{x} \in \boldsymbol{R}^{n}$ the limit

$$
\mathscr{E}^{\infty}(V, C+\bar{x}) \equiv \lim _{t \rightarrow \infty} \mathscr{E}(V, C+\bar{x}, t)
$$

exists and

$$
\mathscr{E}^{\infty}(V, C+\bar{x})-\mathscr{E}^{\infty}(V, C)=\mathscr{E}\left(V_{0}, C+\bar{x}, 0\right)-\mathscr{E}\left(V_{0}, C, 0\right)
$$

For a measurable cone $C$ with its vertex at the origin, we denote by $C_{S_{j, k}}$ the cone

$$
\left\{\alpha s_{j, k}(\theta) \in \boldsymbol{R}^{n} ; \alpha>0, \theta \in C \cap \Theta\right\}
$$

Corollary 1.5. Let $C$ be a measurable cone with the property that

$$
\begin{aligned}
& C_{S_{j, k}} \cap\left(-C_{S_{j, k}}\right)=\emptyset \\
& \quad \text { for any }(j, k) \text { with } 1 \leqq j \leqq M \text { and } 1 \leqq k \leqq m_{j} .
\end{aligned}
$$

Let $h \in L^{2}\left(\boldsymbol{R}^{n}\right)$ be a function such that 


$$
\begin{aligned}
& E_{0}(\xi) \hat{h}(\xi)=0 \quad \text { ae. } \\
& \operatorname{supp} E_{j, k}(\xi) \hat{h}(\xi) \subset(\operatorname{sgn} k) C_{S_{j,|k|} \mid}, \quad 1 \leqq j \leqq M, 1 \leqq|k| \leqq m_{j},
\end{aligned}
$$

where $E_{0}(\xi)$ and $E_{j, k}(\xi)$ is the orthogonal projections onto the eigenspaces of the matrix $\sum_{j=1}^{n} \xi_{j} A_{j}$ corresponding to the eigenvalues 0 and $\lambda_{j, k}(\xi)$ respectively, and $\hat{h}$ is the Fourier transform of $h$.

Then, the solution $V(t, x)$ of (1.1) with the initial value $V(0, x)=h(x)$ is asymptotically focused in the cone $\bar{x}+C$ for any fixed $\bar{x} \in \boldsymbol{R}^{n}$ in the sense that

$$
\mathscr{E}^{\infty}(V, C+\bar{x})=\mathscr{E}\left(V, \boldsymbol{R}^{n}, 0\right)
$$

Moreover, if the $A_{j}$ are real symmetric, then there exist real valued initial data such that (1.23) holds.

The remainder of this paper is organized as follows. $\$ 2$ is devoted to basic studies on wave functions, wave profiles and asymptotic wave functions. The proofs of the results stated in $\S 1$ are given in $\S 3$. $\S 4$ is an Appendix in which we give an algebraic theorem ${ }^{\dagger}$ (see also S. Matuura [5]).

The author would like to express his sincere gratitude to Professors M. Matsumura, H. Suzuki and S. Wakabayashi for their kind advices.

\section{§2. Asymptotic Behavior of Wave Functions}

$$
\boldsymbol{v}_{ \pm}(\boldsymbol{t}, \boldsymbol{x}) \text { for }|\boldsymbol{x}| \longrightarrow \infty
$$

Let $\lambda(\xi)$ be a real valued function on $\boldsymbol{R}^{n}$ satisfying the conditions;

$$
\begin{aligned}
& \lambda \in C^{\infty}\left(\boldsymbol{R}^{n} \backslash\{0\}\right), \\
& \lambda(\xi)>0 \quad \text { for any } \xi \in \boldsymbol{R}^{n} \backslash\{0\} \text { and } \\
& \lambda(\alpha \xi)=\alpha \lambda(\xi) \quad \text { for any } \quad \alpha>0 \text { and any } \xi \in \boldsymbol{R}^{n} \backslash\{0\} .
\end{aligned}
$$

Then the set

$$
S=\left\{s \in \boldsymbol{R}^{n} ; \lambda(s)=1\right\}
$$

\footnotetext{
$\dagger$ We need this theorem in the proof of Corollary 1.3.
} 
forms an $(n-1)$-dimensional closed $C^{\infty}$-manifold embedded in $\boldsymbol{R}^{n}$ which encloses the origin. Let us denote by $K(s)$ the Gaussian curvature of the hypersurface $S$ at each point $s$. Throughout this section we assume

$$
K(s) \neq 0 \quad \text { for any } \quad s \in S \text {. }
$$

Consequently the Gauss map: $S \ni s \mapsto \theta(s) \in \Theta$ is a $C^{\infty}$-diffeomorphism of $S$ onto $\Theta$ where $\Theta$ is the unit sphere in $\boldsymbol{R}^{n}$ and $\theta(s)$ denotes the outward unit normal to $S$ at $s$. The inverse of the Gauss map is denoted by $s(\theta)$ (see for example Sternberg [6]).

Now consider the $C^{\infty}$-map $s^{*}$ :

$$
S \ni s \longmapsto s^{*}(s)=\operatorname{grad} \lambda(s) \in \mathbb{R}^{n} .
$$

Then, it follows from the assumption (2.5) that

$$
S^{*}=\left\{s^{*}(s) ; s \in S\right\}
$$

is an (n-1)-dimensional closed manifold (hypersurface) and the map $s^{*}$ is a $C^{\infty}$-diffeomorphism from $S$ to $S^{*}$. We define the function $\lambda^{*}(x)$ by

$$
\lambda^{*}(x)= \begin{cases}|x| /\left|s^{*}(s(\theta))\right| & \text { for } \quad x=|x| \theta \in \boldsymbol{R}^{n} \backslash\{0\} \\ 0 & \text { for } \quad x=0 .\end{cases}
$$

$\lambda^{*}(x)$ satisfies the conditions (2.1), (2.2) and (2.3), and we have

$$
S^{*}=\left\{s^{*} \in \mathbb{R}^{n} ; \lambda^{*}\left(s^{*}\right)=1\right\}
$$

For each $h_{ \pm} \in L^{2}\left(\boldsymbol{R}^{n}\right)$, we define the function $v_{ \pm}(t, x)$ by

$$
v_{ \pm}(t, x)=\mathscr{F}^{-1}\left[\hat{h}_{ \pm}(\xi) \exp \{\mp i t \lambda( \pm \xi)\}\right](x), \quad i=\sqrt{-1},
$$

where $\mathscr{F}^{-1}[u](x)$ denotes the inverse Fourier transform of $u$ and $\hat{h}$ the Fourier transform of $h . v_{ \pm}(t, \cdot)$ is a $L^{2}\left(\boldsymbol{R}^{n}\right)$-valued solution satisfying the evolution equation

$$
\left(\partial / \partial t \pm i \lambda\left( \pm D_{x}\right)\right) v_{ \pm}(t, x)=0
$$

and the initial condition

$$
v_{ \pm}(0, x)=h_{ \pm}(x)
$$


where $\lambda\left( \pm D_{x}\right)$ is the operator defined by

$$
\lambda\left( \pm D_{x}\right) u(x)=\mathscr{F}^{-1}[\lambda( \pm \xi) \hat{u}(\xi)]
$$

According the fact that $\partial / \partial t \pm i \lambda\left( \pm D_{x}\right)$ is a hyperbolic pseudo-differential operator, we shall call the function $v_{ \pm}(t, x)$ wave function.

For each $H(r, \theta) \in L^{2}(\boldsymbol{R} \times \Theta)$, we define the corresponding function $w_{ \pm}^{\infty}(t, x)$ by

$$
\begin{gathered}
w_{ \pm}^{\infty}(t, x)=H\left(\lambda^{*}(x) \mp t, \theta\right)\left(\lambda^{*}(\theta)\right)^{\frac{1}{2}}|x|^{-(n-1) / 2}, \\
x=|x| \theta \in \boldsymbol{R}^{n} \backslash\{0\} .
\end{gathered}
$$

Then we have

Proposition 2.2. The function $w_{ \pm}^{\infty}(t, x)$ has the properties:

$$
\begin{aligned}
& w_{ \pm}^{\infty}(t, \cdot) \in L^{2}\left(\boldsymbol{R}^{n}\right) \quad \text { for all } t \in \mathbb{R} . \\
& \lim _{t \rightarrow \infty}\left\|w_{-}^{\infty}(t, \cdot)\right\|=0 . \\
& \lim _{t \rightarrow \infty}\left\|w_{+}^{\infty}(t, \cdot)\right\|_{L^{2}(C)}=\|H\|_{L^{2}\left(\boldsymbol{R} \times C_{0}\right)},
\end{aligned}
$$

for any measurable cone $C \subset \mathbb{R}^{n}$ with vertex at the origin where $C_{0}$ $=C \cap \Theta$.

Proof. The definition (2.14) and Fubini's theorem imply (2.15) and

$$
\left\|w_{ \pm}^{\infty}(t, x)\right\|_{L^{2}(C)}^{2}=\int_{C_{0}} d \Theta \int_{0}^{\infty}\left|H\left(r \lambda^{*}(\theta) \mp t, \theta\right)\right|^{2} \lambda^{*}(\theta) d r
$$

Making the change of variables $r \lambda^{*}(\theta) \mp t$ in the second member, we find

$$
\left\|w_{ \pm}^{\infty}(t, x)\right\|_{L^{2}(C)}^{2}=\int_{\mp t}^{\infty} d \rho \int_{C_{0}}|H(\rho, \theta)|^{2} d \Theta
$$

where $d \Theta$ is the surface element on $\Theta$. Letting $t \rightarrow \infty$ in (2.18), we get (2.16) if we take $C=\boldsymbol{R}^{n}$, and (2.17) for general $C$.

Q.E.D.

Definition. For each $h_{ \pm} \in L^{2}\left(\boldsymbol{R}^{n}\right)$ the corresponding wave profile $G_{ \pm} \in L^{2}(\boldsymbol{R} \times \Theta)$ is defined by 


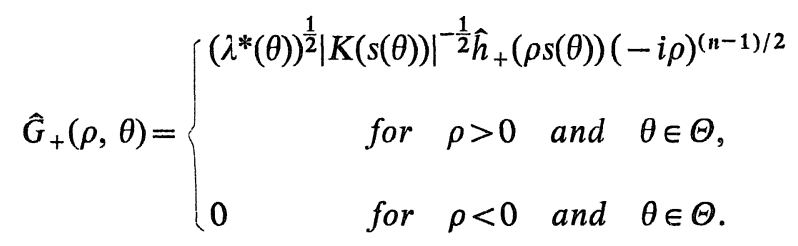

$$
\begin{aligned}
& \tilde{G}_{-}(\rho, \theta)=\left\{\begin{aligned}
&\left(\lambda^{*}(\theta)\right)^{\frac{1}{2}}|K(s(\theta))|^{-\frac{1}{2}} \hat{h}_{-}(-\rho s(\theta))(i \rho)^{(n-1) / 2} \\
& \text { for } \rho>0 \text { and } \theta \in \Theta, \\
& 0 \text { for } \quad \rho<0 \text { and } \theta \in \Theta,
\end{aligned}\right.
\end{aligned}
$$

where for $H(r, \theta) \in L^{2}(\boldsymbol{R} \times \Theta), \hat{H}(\rho, \theta)$ and $\tilde{H}(\rho, \theta)$ denote respectively the partial Fourier transform and the inverse one of $H(r, \theta)$ with respect to $r$, and $( \pm i)^{\frac{1}{2}}=\exp \{ \pm \pi i / 4\}$.

The map

$$
\boldsymbol{R} \times S \ni(\rho, s) \longmapsto(\rho, \theta(s)) \in \boldsymbol{R} \times \Theta
$$

induces an isomorphism of $L^{2}(\boldsymbol{R} \times S)$ onto $L^{2}(\boldsymbol{R} \times \Theta)$ and we have

$$
\int_{R \times C_{0}}|H(\rho, \theta)|^{2} d \rho d \Theta=\int_{R \times s\left(C_{0}\right)}|H(\rho, \theta(s))|^{2}|K(s)| d \rho d S
$$

for any $H \in L^{2}(\boldsymbol{R} \times \Theta)$ and for any measurable subset $C_{0}$ of $\Theta$, where $d S$ is the surface element on $S$.

Proposition 2.3. Let $C_{0}$ be a measurable subset of $\Theta$. Then

$$
\begin{aligned}
& G_{+} \text {and } G_{-} \text {are orthogonal in } L^{2}\left(\boldsymbol{R} \times C_{0}\right) . \\
& \left\|G_{ \pm}\right\|_{L^{2}\left(\boldsymbol{R} \times C_{0}\right)}=\left\|\hat{h}_{ \pm}\right\|_{L^{2}\left( \pm C_{s}\right)},
\end{aligned}
$$

where $C_{S}=\left\{\alpha s(\theta) \in \boldsymbol{R}^{n} ; \alpha>0, \theta \in C_{0}\right\}$.

$$
\text { If } \quad \bar{h}_{+}=h_{-}, \quad \bar{G}_{+}=G_{-} \text {. }
$$

Here, $\bar{h}_{+}$and $\bar{G}_{+}$denote the complex conjugates of $h_{+}$and $G_{+}$respectively.

Proof. (2.23) is obvious since the supports of $\hat{G}_{+}(\rho, \theta)$ and $\hat{G}_{-}(\rho, \theta)$ 
$=\widetilde{G}_{-}(-\rho, \theta)$ are disjoint.

Using Parseval's formula, (2.19), (2.22) and $\lambda^{*}(\theta(s))=|\operatorname{grad} \lambda(s)|^{-1}$, we have

$$
\begin{aligned}
\left\|G_{+}\right\|_{L^{2}\left(\boldsymbol{R} \times C_{0}\right)}^{2} & =\left\|\hat{G}_{+}\right\|_{L^{2}\left(\boldsymbol{R} \times C_{0}\right)}^{2} \\
& =\int_{\boldsymbol{R} \times s\left(C_{0}\right)}\left|\hat{G}_{+}(\rho, \theta(s))\right|^{2}|K(s)| d \rho d S \\
& =\int_{0}^{\infty} \rho^{n-1} d \rho \int_{s\left(C_{0}\right)}\left|\hat{h}_{+}(\rho s)\right|^{2}|\operatorname{grad} \lambda(s)|^{-1} d S
\end{aligned}
$$

In the last integral, let us make the change of variables:

$$
\boldsymbol{R}_{+} \times S \ni(\rho, s) \longmapsto \xi=\rho s \in \boldsymbol{R}^{n} \backslash\{0\} .
$$

Since

$$
d \xi=\rho^{n-1}|\operatorname{grad} \lambda(s)|^{-1} d \rho d S,
$$

we find

$$
\left\|G_{+}\right\|_{L^{2}\left(R \times C_{0}\right)}^{2}=\int_{C_{S}}\left|\hat{h}_{+}(\xi)\right|^{2} d \xi
$$

The corresponding result for $G_{-}$follows by the same argument.

Finally, if $\bar{h}_{+}=h_{-}$, then $\overline{h_{+}(-\xi)}=\hat{h}_{-}(\xi)$. Combining this relation, (2.19) and (2.20), we obtain

$$
\begin{aligned}
\overline{\hat{G}_{+}(-\rho, \theta)} & =\left(\lambda^{*}(\theta)\right)^{\frac{1}{2}}|K(s(\theta))|^{-\frac{1}{2}} \overline{h_{+}(-\rho s(\theta))(i \rho)^{(n-1) / 2}} \\
& =\left(\lambda^{*}(\theta)\right)^{\frac{1}{2}}|K(s(\theta))|^{-\frac{1}{2} \hat{h}_{-}(\rho s(\theta))(-i \rho)^{(n-1) / 2}} \\
& =\widetilde{G}_{-}(-\rho, \theta)=\widehat{G}_{-}(\rho, \theta) \quad \text { for } \quad \rho<0, \theta \in \Theta,
\end{aligned}
$$

and

$$
\overline{\hat{G}_{+}(-\rho, \theta)}=0=\widetilde{G}_{-}(-\rho, \theta)=\hat{G}_{-}(\rho, \theta) \quad \text { for } \quad \rho>0, \theta \in \Theta .
$$

From these relations it follows that $\bar{G}_{+}=G_{-}$.

Q.E.D.

Definition. For the wave function $v_{ \pm}(t, x)$ with $v_{ \pm}(0, x)=h_{ \pm}(x)$, 
we define the corresponding asymptotic wave function $v_{ \pm}^{\infty}(t, x)$ by

$$
\begin{gathered}
v_{ \pm}^{\infty}(t, x)=\left(\lambda^{*}(\theta)\right)^{\frac{1}{2}} G_{ \pm}\left(\lambda^{*}(x)-t, \theta\right)|x|^{-(n-1) / 2}, \\
x=|x| \theta \in \boldsymbol{R}^{n} \backslash\{0\} .
\end{gathered}
$$

This name can be justified by the following theorem.

Theorem 2.4. For $h_{ \pm} \in L^{2}\left(\boldsymbol{R}^{n}\right)$, let $v_{ \pm}(t, x)$ be the solution of the equations (2.11) and (2.12). Let $v_{ \pm}^{\infty}(t, x)$ be the corresponding asymptotic wave function defined by (2.27). Then

$$
\lim _{t \rightarrow \infty}\left\|v_{ \pm}(t, \cdot)-v_{ \pm}^{\infty}(t, \cdot)\right\|=0
$$

Proof. Since $C_{0}^{\infty}\left(\boldsymbol{R}^{n} \backslash\{0\}\right)$ is dense in $L^{2}\left(\boldsymbol{R}^{n}\right)$, it is sufficient to prove the theorem for functions $h_{ \pm} \in L^{2}\left(\boldsymbol{R}^{n}\right)$ such that $\hat{h}_{ \pm} \in C_{0}^{\infty}\left(\boldsymbol{R}^{n} \backslash\{0\}\right)$. Thus we may assume that

$$
\operatorname{supp} \hat{h}_{ \pm} \subset\left\{\xi \in \boldsymbol{R}^{n} ; 0<a \leqq \lambda( \pm \xi) \leqq b<\infty\right\} .
$$

From (2.10) we have

$$
\begin{aligned}
v_{ \pm}(t, x) & =(2 \pi)^{-n / 2} \int_{a \leqq \lambda( \pm \xi) \leqq b} \hat{h}_{ \pm}(\xi) \exp \{i x \xi \mp i t \lambda( \pm \xi)\} d \xi \\
& =(2 \pi)^{-n / 2} \int_{a \leqq \lambda(\xi) \leqq b} \hat{h}_{ \pm}( \pm \xi) \exp \{ \pm i x \xi \mp i t \lambda(\xi)\} d \xi
\end{aligned}
$$

Transforming to the polar coordinates $(\rho, s)$ with respect to $S$ (or $\lambda(\xi)$ ), we get

$$
\begin{aligned}
v_{ \pm}(t, x)= & (2 \pi)^{-n / 2} \int_{a}^{b} \rho^{n-1} \exp \{\mp i t \rho\} d \rho \\
& \times \int_{S} \hat{h}_{ \pm}( \pm \rho s) \exp \{ \pm i \rho x s\}\left|s^{*}(s)\right|^{-1} d S
\end{aligned}
$$

Let us set

$$
\omega_{ \pm}(x, \rho)=\int_{S} \hat{h}_{ \pm}( \pm \rho s) \exp \{ \pm i \rho x s\}\left|s^{*}(s)\right|^{-1} d S .
$$

Applying the method of stationary phase to this integral, we find 
(2.32)

$$
\begin{aligned}
& \omega_{ \pm}(x, \rho)=(2 \pi /|\rho x|)^{(n-1) / 2} \\
& \times\left[\frac{\hat{h}_{ \pm}( \pm \rho s( \pm \theta))}{|K(s( \pm \theta))|^{\frac{1}{2}}\left|s^{*}(s( \pm \theta))\right|} \exp \{-i \pi(n-1) / 4 \pm i \rho x s( \pm \theta)\}\right. \\
& \left.+\frac{\hat{h}_{ \pm}( \pm \rho s(\mp \theta))}{|K(s(\mp \theta))|^{\frac{1}{2}}\left|s^{*}(s(\mp \theta))\right|} \exp \{i \pi(n-1) / 4 \pm i \rho x s(\mp \theta)\}\right] \\
& \quad+q(x, \rho)
\end{aligned}
$$

where the function $q(x, \rho)$ satisfies

(2.33) $|q(x, \rho)| \leqq M|x|^{-(n+1) / 2} \quad$ for all $x \in \boldsymbol{R}^{n} \backslash\{0\}$ and $\rho \in[a, b]$,

$M$ being a positive constant independent of $x$ and $\rho \in[a, b]$, but dependent on $a$ and $b$. For the method of stationary phase, see, for example, Littman [2] or Matsumura [4] (Theorem 5.1). Substituting (2.32) into (2.30) and using the relation $\lambda^{*}( \pm \theta)=\left|s^{*}(s( \pm \theta))\right|^{-1}$, we obtain

$$
\begin{aligned}
v_{ \pm}(t, x)= & \left(\lambda^{*}(\theta) / 2 \pi\right)^{\frac{1}{2}}|x|^{-(n-1) / 2} \int_{a}^{b}\left(\lambda^{*}(\theta) /|K(s(\theta))|\right)^{\frac{1}{2}} \\
& \times \hat{h}_{ \pm}( \pm \rho s(\theta))(\mp i \rho)^{(n-1) / 2} \exp \{ \pm i \rho(x s(\theta)-t)\} d \rho \\
& +\left(\lambda^{*}(-\theta) / 2 \pi\right)^{\frac{1}{2}}|x|^{-(n-1) / 2} \int_{a}^{b}\left(\lambda^{*}(-\theta) /|K(s(-\theta))|\right)^{\frac{1}{2}} \\
& \times \hat{h}_{ \pm}( \pm \rho s(-\theta))( \pm i \rho)^{(n-1) / 2} \exp \{ \pm i \rho(x s(-\theta)-t)\} d \rho \\
& +(2 \pi)^{-n / 2} \int_{a}^{b} q(x, \rho) \rho^{n-1} \exp \{\mp i \rho t\} d \rho .
\end{aligned}
$$

Now, we have

$$
\begin{aligned}
x s( \pm \theta) & =|x| \theta s( \pm \theta) \\
& =|x|( \pm \operatorname{grad} \lambda(s( \pm \theta))) s( \pm \theta) /\left|s^{*}(s( \pm \theta))\right| \\
& = \pm|x| \lambda(s( \pm \theta)) /\left|s^{*}(s( \pm \theta))\right|= \pm \lambda^{*}( \pm x),
\end{aligned}
$$

since $s \cdot \operatorname{grad} \lambda(s)=\lambda(s)=1$ for $s \in S$. Consequently,

$$
v_{ \pm}(t, x)=\left(\lambda^{*}(\theta) / 2 \pi\right)^{\frac{1}{2}}|x|^{-(n-1) / 2} \int_{a}^{b}\left(\lambda^{*}(\theta) /|K(s(\theta))|\right)^{\frac{1}{2}}
$$




$$
\begin{aligned}
& \times \hat{h}_{ \pm}( \pm \rho s(\theta))(\mp i \rho)^{(n-1) / 2} \exp \left\{ \pm i \rho\left(\lambda^{*}(x)-t\right)\right\} d \rho \\
& +\left(\lambda^{*}(-\theta) / 2 \pi\right)^{\frac{1}{2}}|x|^{-(n-1) / 2} \int_{a}^{b}\left(\lambda^{*}(-\theta) /|K(s(-\theta))|\right)^{\frac{1}{2}} \\
& \times \hat{h}_{ \pm}( \pm \rho s(-\theta))( \pm i \rho)^{(n-1) / 2} \exp \left\{\mp i \rho\left(\lambda^{*}(-x)+t\right)\right\} d \rho \\
& +(2 \pi)^{-n / 2} \int_{a}^{b} q(x, \rho) \rho^{n-1} \exp \{\mp i \rho t\} d \rho .
\end{aligned}
$$

Since $\hat{h}_{ \pm}$satisfies (2.29), the first term on the right in (2.36) is exactly the asymptotic wave function $v_{ \pm}^{\infty}(t, x)$. Hence, in order to complete the proof we only need to show that two remaining terms tend to zero in $L^{2}\left(\boldsymbol{R}^{n}\right)$ when $t \rightarrow \infty$. For the second term on the right in (2.36), it follows from (2.16). Consider the third term. By (2.32) the function $q(x, \rho)$ has the order $|x|^{-(n-1) / 2}$ uniformly in $\rho \in[a, b]$ and in $\theta \in \Theta$ when $|x| \rightarrow 0$ and has the order $|x|^{-(n+1) / 2}$ by (2.33) when $|x| \rightarrow \infty$.

Consequently there exists a function $q_{+}(x) \in L^{2}\left(\boldsymbol{R}^{n}\right)$ such that

$$
|q(x, \rho)| \leqq q_{+}(x) \quad \text { for any } \rho \in[a, b] .
$$

Then we have

$$
\left|\int_{a}^{b} q(x, \rho) \rho^{n-1} \exp \{\mp i \rho t\} d \rho\right|^{2} \leqq\left(\int_{a}^{b} \rho^{n-1} d \rho\right)^{2}\left|q_{+}(x)\right|^{2}
$$

If $x$ is fixed, the third term on the right of (2.36) tends to zero when $t \rightarrow \infty$ by Riemann-Lebesgue Theorem. Since (2.38) holds, it also converges to zero in $L^{2}\left(\boldsymbol{R}^{n}\right)$ when $t \rightarrow \infty$ by Lebesgue's Theorem of dominated convergence.

Q.E.D.

Let $\theta_{1}(t)$ and $\theta_{2}(t)$ be two real valued functions of $t>0$ that satisfy

$$
-t \leqq \theta_{1}(t) \leqq \theta_{2}(t) \quad \text { for all } \quad t>0 .
$$

Denote by $B\left(t, \theta_{1}(t), \theta_{2}(t)\right)$ the expanding spherical zone of the form

$$
\left\{x \in \boldsymbol{R}^{n} ; t+\theta_{1}(t) \leqq \lambda^{*}(x) \leqq t+\theta_{2}(t)\right\} .
$$

Then we have

\section{Proposition 2.5.}


(2.41) $\quad \mathscr{E}\left(v_{ \pm}, B\left(t, \theta_{1}(t), \theta_{2}(t)\right), t\right)=\int_{\theta_{1}(t)}^{\theta_{2}(t)}\left\|G_{ \pm}(r, \cdot)\right\|_{L^{2}(\Theta)}^{2} d r+o(1)$,

where $o(1)$ denotes a function of $t$ which tends to zero for $t \rightarrow \infty$, uniformly in $\theta_{1}(t), \theta_{2}(t)$.

Proof. Note that

$$
\left\|v_{ \pm}^{\infty}(t, \cdot)\right\|_{L^{2}\left(B\left(t, \theta_{1}(t), \theta_{2}(t)\right)\right)}^{2}=\int_{\theta_{1}(t)}^{\theta_{2}(t)} d r \int_{\Theta}\left|G_{ \pm}(r, \theta)\right|^{2} d \Theta
$$

and

$$
\left|\left\|v_{ \pm}(t, \cdot)\right\|_{L^{2}(B)}-\left\|v_{ \pm}^{\infty}(t, \cdot)\right\|_{L^{2}(B)}\right| \leqq\left\|v_{ \pm}(t, \cdot)-v_{ \pm}^{\infty}(t, \cdot)\right\|
$$

for any measurable subset $B$ of $\boldsymbol{R}^{n}$. Combining (2.42), (2.43) and (2.28), we get (2.41).

Q.E.D.

Corollary 2.6. Let $\theta_{1}(t)$ and $\theta_{2}(t)$ satisfy the conditions (2.39) and

$$
\lim _{t \rightarrow \infty} \theta_{1}(t)=-\infty, \lim _{t \rightarrow \infty} \theta_{2}(t)=\infty
$$

Then we have

$$
\lim _{t \rightarrow \infty} \mathscr{E}\left(v_{ \pm}, \boldsymbol{R}^{n} \backslash B\left(t, \theta_{1}(t), \theta_{2}(t)\right), t\right)=0
$$

Proof. Taking $C_{0}=\Theta$ in (2.24), we have

$$
\left\|G_{ \pm}\right\|_{L^{2}(\boldsymbol{R} \times \Theta)}=\left\|\hat{h}_{ \pm}\right\|=\left\|h_{ \pm}\right\| \text {。 }
$$

On the other hand

$$
\mathscr{E}\left(v_{ \pm}, \boldsymbol{R}^{n}, t\right)=\mathscr{E}\left(v_{ \pm}, \boldsymbol{R}^{n}, 0\right)=\left\|h_{ \pm}\right\|^{2}
$$

Combining (2.41), (2.46) and (2.47), we get

$$
\begin{aligned}
\mathscr{E}\left(v_{ \pm}, \boldsymbol{R}^{n} \backslash B\left(t, \theta_{1}(t), \theta_{2}(t)\right), t\right) \\
=\quad \mathscr{E}\left(v_{ \pm}, \boldsymbol{R}^{n}, t\right)-\mathscr{E}\left(v_{ \pm}, B\left(t, \theta_{1}(t), \theta_{2}(t)\right), t\right) \\
\quad=\left\|h_{ \pm}\right\|^{2}-\int_{\theta_{1}(t)}^{\theta_{2}(t)}\left\|G_{ \pm}(r, \cdot)\right\|_{L^{2}(\theta)}^{2} d r+o(1)
\end{aligned}
$$




$$
\begin{aligned}
& =\int_{-\infty}^{\theta_{1}(t)}\left\|G_{ \pm}(r, \cdot)\right\|_{L^{2}(\Theta)}^{2} d r+\int_{\theta_{2}(t)}^{\infty}\left\|G_{ \pm}(r, \cdot)\right\|_{L^{2}(\Theta)}^{2} d r+o(1) \\
& =o(1) \quad t \rightarrow \infty \text { by hypotheses }(2.44)
\end{aligned}
$$

Corollary 2.7. For any bounded measurable subset $K$ of $\boldsymbol{R}^{n}$

$$
\lim _{t \rightarrow \infty} \mathscr{E}\left(v_{ \pm}, K, t\right)=0
$$

Corollary 2.8. For any $\varepsilon>0$ there exist constants $\theta_{1}=\theta_{1}\left(h_{ \pm}, \varepsilon\right)$, $\theta_{2}=\theta_{2}\left(h_{ \pm}, \varepsilon\right)$ and $t_{0}=t_{0}\left(h_{ \pm}, \varepsilon\right)$ such that

$$
\begin{gathered}
\mathscr{E}\left(v_{ \pm}, \boldsymbol{R}^{n}, 0\right)-\varepsilon \leqq \mathscr{E}\left(v_{ \pm}, B\left(t, \theta_{1}, \theta_{2}\right), t\right) \\
\leqq \mathscr{E}\left(v_{ \pm}, \boldsymbol{R}^{n}, 0\right) \quad \text { for all } t \geqq t_{0}
\end{gathered}
$$

Proof. For any functions $\theta_{1}(t)$ and $\theta_{2}(t)$ with the property (2.39) we have

$$
\begin{aligned}
& \mathscr{E}\left(v_{ \pm}, \boldsymbol{R}^{n}, 0\right)-\mathscr{E}\left(v_{ \pm}, B\left(t, \theta_{1}(t), \theta_{2}(t)\right), t\right) \\
& =\int_{-\infty}^{\infty}\left\|G_{ \pm}(r, \cdot)\right\|_{L^{2}(\Theta)}^{2} d r-\mathscr{E}\left(v_{ \pm}, B\left(t, \theta_{1}(t), \theta_{2}(t)\right), t\right) \\
& \leqq \int_{-\infty}^{\theta_{1}(t)}\left\|G_{ \pm}(r, \cdot)\right\|_{L^{2}(\Theta)}^{2} d r+\int_{\theta_{2}(t)}^{\infty}\left\|G_{ \pm}(r, \cdot)\right\|_{L^{2}(\Theta)}^{2} d r \\
& \quad+\left|\int_{\theta_{1}(t)}^{\theta_{2}(t)}\left\|G_{ \pm}(r, \cdot)\right\|_{L^{2}(\theta)}^{2} d r-\mathscr{E}\left(v_{ \pm}, B\left(t, \theta_{1}(t), \theta_{2}(t)\right), t\right)\right|
\end{aligned}
$$

for all $t>0$. Now Proposition 2.5 implies that there exists a constant $t_{0}=t_{0}\left(h_{ \pm}, \varepsilon\right)$, independent of $\theta_{1}(t)$ and $\theta_{2}(t)$ such that the last term in (2.50) is less than $\varepsilon / 2$ for all $t \geqq t_{0}$. Moreover, there exist constants $\theta_{1}=\theta_{1}\left(h_{ \pm}, \varepsilon\right)<0$ and $\theta_{2}=\theta_{2}\left(h_{ \pm}, \varepsilon\right)>0$ such that

$$
\int_{-\infty}^{\theta_{1}}\left\|G_{ \pm}(r, \cdot)\right\|_{L^{2}(\Theta)}^{2} d r+\int_{\theta_{2}}^{\infty}\left\|G_{ \pm}(r, \cdot)\right\|_{L^{2}(\theta)}^{2} d r \leqq \varepsilon / 2
$$

Here we may assume that $t_{0} \geqq \max \left(-\theta_{1}, \theta_{2}\right)$. Therefore, putting

$$
\theta_{1}(t)=\left\{\begin{array}{ll}
0 & \text { for } t<t_{0} \\
\theta_{1} & \text { for } t \geqq t_{0}
\end{array}, \quad \theta_{2}(t)=\left\{\begin{array}{ll}
0 & \text { for } t<t_{0} \\
\theta_{2} & \text { for } t \geqq t_{0}
\end{array}\right. \text {, }\right.
$$


we get (2.49).

Q.E.D.

Proposition 2.9. For any measurable cone $C$ of $\boldsymbol{R}^{n}$ with vertex at the origin, there exist

$$
\begin{aligned}
& \mathscr{E}^{\infty}\left(v_{ \pm}, C\right) \equiv \lim _{t \rightarrow \infty} \mathscr{E}\left(v_{ \pm}, C, t\right) \\
& \mathscr{E}^{\infty}\left(v_{+}+v_{-}, C\right) \equiv \lim _{t \rightarrow \infty} \mathscr{E}\left(v_{+}+v_{-}, C, t\right)
\end{aligned}
$$

and

$$
\begin{aligned}
& \mathscr{E}^{\infty}\left(v_{ \pm}, C\right)=\left\|\hat{h}_{ \pm}\right\|_{L^{2}\left( \pm c_{s}\right)}^{2} \\
& \mathscr{E}^{\infty}\left(v_{+}+v_{-}, C\right)=\mathscr{E}^{\infty}\left(v_{+}, C\right)+\mathscr{E}^{\infty}\left(v_{-}, C\right),
\end{aligned}
$$

where $C_{S}=\left\{\alpha s(\theta) \in \boldsymbol{R}^{n} ; \alpha>0, \theta \in C \cap \Theta\right\}$.

Proof. Note that

$$
\begin{array}{ll}
\text { (2.55) } & \left|\left\|v_{ \pm}(t, \cdot)\right\|_{L^{2}(C)}-\left\|v_{ \pm}^{\infty}(t, \cdot)\right\|_{L^{2}(C)}\right| \\
& \leqq\left\|v_{ \pm}(t, \cdot)-v_{ \pm}^{\infty}(t, \cdot)\right\|, \\
\text { (2.56) } & \mathscr{E}\left(v_{ \pm}^{\infty}, C, t\right)=\int_{-t}^{\infty}\left\|G_{ \pm}(r, \cdot)\right\|_{L^{2}\left(C_{0}\right)}^{2} d r \\
\text { (2.57) } & \mathscr{E}\left(v_{+}^{\infty}+v_{-}^{\infty}, C, t\right)=\int_{-t}^{\infty}\left\|G_{+}(r, \cdot)+G_{-}(r, \cdot)\right\|_{L^{2}\left(C_{0}\right)}^{2} d r
\end{array}
$$

where $C_{0}=C \cap \Theta$. Combining (2.55), (2.56), (2.57) and (2.28) we get (2.51) and (2.52). Letting $t \rightarrow \infty$ in (2.55), (2.56) and (2.57), we find

$$
\mathscr{E}^{\infty}\left(v_{ \pm}, C\right)=\left\|G_{ \pm}\right\|_{L^{2}\left(R \times C_{0}\right)}^{2}
$$

and

$$
\mathscr{E}^{\infty}\left(v_{+}+v_{-}, C\right)=\left\|G_{+}+G_{-}\right\|_{L^{2}\left(R \times C_{0}\right)}^{2}
$$

Combining (2.58), (2.59), (2.23) and (2.24), we obtain (2.53) and (2.54).

Q.E.D. 


\section{§3. Proof of Results on Symmetric Hyperbolic Systems}

Consider the operator $L=I(\partial / \partial t)+\sum_{j=1}^{n} A_{j}\left(\partial / \partial x_{j}\right)$ satisfying the conditions (L.1) and (L.2) in $\S 1$. The roots 0 and $\lambda_{j, k}(\xi), j=1, \ldots, M,|k|$ $=1, \ldots, m_{j}$ of the equation $P(\lambda, \xi)=0$ in $\lambda$ are eigenvalues of the matrix $A(\xi)=\sum_{j=1}^{n} \xi_{j} A_{j}$. Let us denote by $E_{0}(\xi)$ and $E_{j, k}(\xi)$ the orthogonal projections $^{\dagger}$ onto the eigenspaces corresponding to the eigenvalues 0 and $\lambda_{j, k}(\xi)$, respectively. As is well known, $E_{0}(\xi)$ and $E_{j, k}(\xi)$ can be represented respectively in the form

$$
E_{0}(\xi)=(2 \pi i)^{-1} \int_{\Gamma_{0}(\xi)}(\zeta I-A(\xi))^{-1} d \zeta
$$

and

$$
E_{j, k}(\xi)=(2 \pi i)^{-1} \int_{\Gamma_{j, k}(\xi)}(\zeta I-A(\xi))^{-1} d \zeta
$$

where $\Gamma_{0}(\xi)$ and $\Gamma_{j, k}(\xi)$ are positively oriented circles which encloses the eigenvalues 0 and $\lambda_{j, k}(\xi)$, respectively, but no other different eigenvalues. The matrix valued functions $E_{0}(\xi)$ and $E_{j, k}(\xi)$ are measurable and positively homogeneous of degree zero. Further they have the following properties which are valid for almost all $\xi \in \mathbb{R}^{n}$.

$$
\begin{array}{ll}
\text { (3.3) } & E_{0}^{2}(\xi)=E_{0}(\xi), \quad E_{j, k}^{2}(\xi)=E_{j, k}(\xi) \\
& E_{0}(\xi) E_{j, k}(\xi)=E_{j, k}(\xi) E_{0}(\xi)=0 \text { and } \\
& E_{j, k}(\xi) E_{j^{\prime}, k^{\prime}}(\xi)=E_{j^{\prime}, k^{\prime}}(\xi) E_{j, k}(\xi)=0 \text { if }(j, k) \neq\left(j^{\prime}, k^{\prime}\right) . \\
\text { (3.4) } & E_{0}^{*}(\xi)=E_{0}(\xi) \text { and } E_{j, k}^{*}(\xi)=E_{j, k}(\xi) \\
\text { (3.5) } & E_{0}(\xi)+\sum_{j=1}^{M} \sum_{|k|=1}^{m_{j}} E_{j, k}(\xi)=I .
\end{array}
$$

For a solution $V(t, \cdot) \in L^{2}\left(\boldsymbol{R}^{n}\right)$ of the equations (1.1) and (1.2), we put

$$
\hat{V}_{0}(t, \xi)=E_{0}(\xi) \hat{V}(t, \xi), \quad \widehat{V}_{j, k}(t, \xi)=E_{j, k}(\xi) \hat{V}(t, \xi)
$$

$\dagger$ These projections can be defined for almost all $\xi \in \boldsymbol{R}^{n}$. 
(3.7) $\quad \hat{h}_{0}(\xi)=E_{0}(\xi) \hat{h}(\xi)$ and $\hat{h}_{j, k}(\xi)=E_{j, k}(\xi) \hat{h}(\xi)$.

Then we have

(3.8) $\quad V(t, x)=V_{0}(t, x)+\sum_{j=1}^{M} \sum_{|k|=1}^{m_{j}} V_{j, k}(t, x)$,

(3.9) $\quad \frac{\partial \hat{V}_{0}}{\partial t}(t, \xi)=0, \quad \hat{\nabla}_{0}(0, \xi)=\hat{h}_{0}(\xi)$

(3.10) $\frac{\partial \hat{V}_{j, k}}{\partial t}(t, \xi)=\mp i \lambda_{j, k}( \pm \xi) \hat{V}_{j, k}(t, \xi), \quad \hat{V}_{j, k}(0, \xi)=\hat{h}_{j, k}(\xi)$

The asymptotic wave profile $G_{j, k}$ corresponding to each $h_{j, k}$ is defined by

$$
\hat{G}_{j, k}(\rho, \theta)=\left\{\begin{array}{r}
\left(\lambda_{j, k}^{*}(\theta) /\left|K_{j, k}\left(s_{j, k}(\theta)\right)\right|\right)^{\frac{1}{2}} \\
\times \hat{h}_{j, k}\left(\rho s_{j, k}(\theta)\right)(-i \rho)^{(n-1) / 2} \\
\text { for } \rho>0 \text { and } \theta \in \Theta \\
0 \quad \text { for } \rho<0 \text { and } \theta \in \Theta
\end{array}\right.
$$

and

$$
\tilde{G}_{j, k}(\rho, \theta)=\left\{\begin{array}{r}
\left(\lambda_{j,|k|}^{*}(\theta) /\left|K_{j,|k|}\left(s_{j,|k|}(\theta)\right)\right|\right)^{\frac{1}{2}} \\
\times \hat{h}_{j, k}\left(-\rho s_{j,|k|}(\theta)\right)(i \rho)^{(n-1) / 2} \\
\text { for } \rho>0 \text { and } \theta \in \Theta \\
0 \quad \text { for } \rho<0 \text { and } \theta \in \Theta
\end{array}\right.
$$

Then the asymptotic wave function $V_{j, k}^{\infty}(t, x)$ corresponding to $V_{j, k}(t, x)$ is defined by (1.15).

Proof of Theorem 1.1. If $V^{\infty}(t, x)$ is the function defined by (1.14), we have

$$
\left\|V(t, \cdot)-V_{0}(\cdot)-V^{\infty}(t, \cdot)\right\| \leqq \sum_{j=1}^{M} \sum_{|k|=1}^{m_{j}}\left\|V_{j, k}(t, \cdot)-V_{j, k}^{\infty}(t, \cdot)\right\| .
$$


Applying Theorem 2.4 to $V_{j, k}$ and $V_{j, k}^{\infty}$, we get (1.13). If the matrices $A_{j}(j=1, \ldots, n)$ are real symmetric and if $h(x)$ is $\boldsymbol{R}^{N}$-valued function, then we have

$$
\overline{E_{0}(-\xi)}=E_{0}(\xi) \text { and } \overline{E_{j, k}(-\xi)}=E_{j,-k}(\xi)
$$

Therefore,

$$
\overline{\hat{V}_{0}(-\xi)}=\hat{V}_{0}(\xi) \text { and } \overline{\hat{h}_{j, k}(-\xi)}=\hat{h}_{j,-k}(\xi)
$$

Applying (2.25) of Proposition 2.3 to these functions, we see that $V_{0}(x)$ and $V^{\infty}(t, x)$ are real valued.

Q.E.D.

Proposition 3.1. Let $\theta_{1}(t)$ and $\theta_{2}(t)$ be two real valued functions of $t>0$ which satisfy (2.39) and (2.44). If we put

$$
B_{j, k}(t)=\left\{x \in \boldsymbol{R}^{n} ; t+\theta_{1}(t) \leqq \lambda_{j, k}^{*}(x) \leqq t+\theta_{2}(t)\right\}
$$

and

$$
B(t)=\bigcup_{j=1}^{M} \bigcup_{k=1}^{m_{j}} B_{j, k}(t),
$$

we have

$$
\lim _{t \rightarrow \infty} \mathscr{E}\left(V-V_{0}, \boldsymbol{R}^{n} \backslash B(t), t\right)=0
$$

Proof. There exist a constant $\alpha$ such that

$$
\begin{aligned}
& \mathscr{E}\left(V-V_{0}, \boldsymbol{R}^{n} \backslash B(t), t\right) \\
& \quad \leqq \alpha \sum_{j=1}^{M} \sum_{|k|=1}^{m_{j}} \mathscr{E}\left(V_{j, k}, \boldsymbol{R}^{n} \backslash B(t), t\right) .
\end{aligned}
$$

On the other hand, we have

$$
\begin{aligned}
\mathscr{E} & \left(V_{j, k}, \boldsymbol{R}^{n} \mid B(t), t\right) \\
& =\mathscr{E}\left(V_{j, k}, \boldsymbol{R}^{n}, t\right)-\mathscr{E}\left(V_{j, k}, B(t), t\right) \\
& \leqq \\
& =\mathscr{E}\left(V_{j, k}, \boldsymbol{R}^{n}, t\right)-\mathscr{E}\left(V_{j, k}, B_{j,|k|}(t), t\right) \\
& =\mathscr{E}\left(V_{j, k}, \boldsymbol{R}^{n} \backslash B_{j,|k|}(t), t\right)
\end{aligned}
$$


Combining (3.18) and (3.19) and applying Corollary 2.6 to each $\mathscr{E}\left(V_{j, k}\right.$, $\left.\boldsymbol{R}^{n} \backslash B_{j,|k|}(t), t\right)$, we get (3.17).

Q.E.D.

Proof of Corollary 1.2. From Proposition 3.1 it follows that the right hand side of the inequality

$$
\left|\sqrt{\mathscr{E}(V, K, t)}-\sqrt{\mathscr{E}\left(V_{0}, K, t\right)}\right| \leqq \sqrt{\mathscr{E}\left(V-V_{0}, K, t\right)}
$$

tends to zero when $t \rightarrow \infty$ for any bounded measurable subset $K$ of $\boldsymbol{R}^{n}$. This means (1.16).

Q.E.D.

Lemma 3.2. If $f \in L^{p}\left(\boldsymbol{R}^{n}\right), g \in L^{q}\left(\boldsymbol{R}^{n}\right)(1 / p+1 / q=1, p>1, q>1)$ and if $T_{1}, T_{2}$ and $T_{1}-T_{2}$ are non singular linear transformations in the vector space $\boldsymbol{R}^{n}$, we have

$$
\lim _{|\zeta| \rightarrow \infty} \int_{\mathbb{R}^{n}} f(\eta) g\left(\eta-T_{1} \zeta\right) d \eta=0
$$

and

$$
\lim _{|\zeta| \rightarrow \infty} \int_{R^{n}} f\left(T_{1} \eta-\zeta\right) g\left(T_{2} \eta-\zeta\right) d \eta=0
$$

Proof. Given $\varepsilon>0$, we choose a compact subset $K$ of $\boldsymbol{R}^{n}$ so that

$$
\left\{\int_{\mathbb{R}^{n} \backslash K}|f(\eta)|^{p} d \eta\right\}^{1 / p} \leqq \varepsilon \text { and }\left\{\int_{R^{n} \backslash K}|g(\eta)|^{q} d \eta\right\}^{1 / q} \leqq \varepsilon
$$

If we put $K(\zeta)=K-T_{1} \zeta$ for $\zeta \in \boldsymbol{R}^{n}$, then there exist $t_{0}>0$ such that

$$
K \cap K(\zeta)=\varnothing, \quad \text { that is, } K(\zeta) \subset \boldsymbol{R}^{n} \backslash K
$$

$$
\text { for all } \zeta \text { with }|\zeta| \geqq t_{0} \text {. }
$$

Consider

$$
\begin{aligned}
\int_{R^{n}} & \left|f(\eta) g\left(\eta-T_{1} \zeta\right)\right| d \eta \\
& =\int_{K} \prime \prime+\int_{K(\zeta)} \prime \prime+\int_{R^{n} \backslash(K \cup K(\zeta))} \prime \prime \\
& =I_{1}+I_{2}+I_{3} \text { respectively. }
\end{aligned}
$$


By Hölder's inequality,

$$
\begin{aligned}
I_{1} & \leqq\left\{\int_{K}|f(\eta)|^{p} d \eta\right\}^{1 / p}\left\{\int_{K}\left|g\left(\eta-T_{1} \zeta\right)\right|^{q} d \eta\right\}^{1 / q} \\
& \leqq\|f\|_{p}\left\{\int_{K(\zeta)}|g(\eta)|^{q} d \eta\right\}^{1 / q} \leqq \varepsilon\|f\|_{p} .
\end{aligned}
$$

Similarly

$$
I_{2} \leqq \varepsilon\|g\|_{q}
$$

and

$$
\begin{aligned}
I_{3} & \leqq\left\{\int_{R^{n} \backslash K}|f(\eta)|^{p} d \eta\right\}^{1 / p}\left\{\int_{R^{n}}|g(\eta)|^{q} d \eta\right\}^{1 / q} \\
& \leqq \varepsilon\|g\|_{q^{\prime}}
\end{aligned}
$$

where $\|\cdot\|_{r}$ denotes $L^{r}\left(\boldsymbol{R}^{n}\right)$ norm. Thus we get for any $\zeta$ with $|\zeta| \geqq t_{0}$

$$
\left|\int_{\boldsymbol{R}^{n}} f(\eta) g\left(\eta-T_{1} \zeta\right) d \eta\right| \leqq \varepsilon\left(\|f\|_{p}+2\|g\|_{q}\right)
$$

This means (3.20). In order to show (3.21), let us make the change of variables: $\eta \mapsto \xi=T_{2}\left(\eta-T_{1}^{-1} \zeta\right)$. Then

$$
\begin{aligned}
& \int_{\boldsymbol{R}^{n}} f\left(T_{1} \eta-\zeta\right) g\left(T_{2} \eta-\zeta\right) d \eta \\
& \quad=\left|\operatorname{det}\left(T_{2}\right)\right|^{-1} \int_{\boldsymbol{R}^{n}} f\left(T_{1} T_{2}^{-1} \xi\right) g\left(\xi-\left(I-T_{2} T_{1}^{-1}\right) \zeta\right) d \xi
\end{aligned}
$$

Noting that $f_{1}(\xi)=f\left(T_{1} T_{2}^{-1} \xi\right) \in L^{p}\left(\boldsymbol{R}^{n}\right)$ and applying (3.20) to the integral on the right, we obtain (3.21).

Q.E.D.

Remark. We use this lemma only in one dimensional case $n=1$.

Proof of Corollary 1.3. Consider

$$
\begin{aligned}
& \mathscr{E}\left(V_{0}+V^{\infty}, C, t\right)=\int_{C}\left|V_{0}+\sum_{j=1}^{M} \sum_{|k|=1}^{m_{j}} V_{j, k}^{\infty}\right|^{2} d x \\
& =\mathscr{E}\left(V_{0}, C, t\right)+
\end{aligned}
$$




$$
\begin{aligned}
& +\sum_{j=1}^{M} \sum_{|k|=1}^{m_{j}}\left\{\mathscr{E}\left(V_{j, k}^{\infty}, C, t\right)+\int_{C}\left(V_{0} \cdot V_{j, k}^{\infty}+V_{j, k}^{\infty} \cdot V_{0}\right) d x\right\} \\
& +\sum_{(j, k) \neq\left(j^{\prime}, k^{\prime}\right)}^{\infty} \int_{C} V_{j, k}^{\infty} \cdot V_{j^{\prime}, k^{\prime}}^{\infty} d x,
\end{aligned}
$$

where for $\zeta, z \in C^{N}, \zeta \cdot z$ denotes the inner product $\zeta_{1} \bar{z}_{1}+\cdots+\zeta_{N} \bar{z}_{N}$. We shall show that

$$
\lim _{t \rightarrow \infty} \int_{C}\left|V_{j, k}^{\infty}(t, x) \cdot V_{j^{\prime}, k^{\prime}}^{\infty}(t, x)\right| d x=0, \quad \text { if } \quad(j, k) \neq\left(j^{\prime}, k^{\prime}\right)
$$

and

$$
\lim _{t \rightarrow \infty} \int_{C}\left|V_{0}(x) \cdot V_{j, k}^{\infty}(t, x)\right| d x=0
$$

From (1.15) we have

$$
\begin{aligned}
& \int_{C}\left|V_{j, k}^{\infty}(t, x) \cdot V_{j^{\prime}, k^{\prime}}^{\infty}(t, x)\right| d x \\
& =\int_{C_{0}}\left(\lambda_{j,|k|}^{*}(\theta) \lambda_{j^{\prime},\left|k^{\prime}\right|}^{*}(\theta)\right)^{\frac{1}{2}} d \Theta \\
& \quad \times \int_{0}^{\infty}\left|G_{j, k}\left(\lambda_{j,|k|}^{*}(\theta) r-t, \theta\right) \cdot G_{j^{\prime}, k^{\prime}}\left(\lambda_{j^{\prime},\left|k^{\prime}\right|}^{*}(\theta) r-t, \theta\right)\right| d r
\end{aligned}
$$

where $C_{0}=C \cap \Theta$. Since $G_{j, k}$ and $G_{j,-k}$ are orthogonal in $L^{2}\left(\boldsymbol{R} \times C_{0}\right)$ by (2.23) of Proposition 2.3, this quantity tends to 0 when $t \rightarrow \infty$ if $j=j^{\prime}$ and $k^{\prime}=-k$. For the cases where $j \neq j^{\prime}$, or $j=j^{\prime}$ and $k^{\prime} \neq \pm k$,

$$
\lambda_{j,|k|}^{*}(\theta) \neq \lambda_{j^{\prime},\left|k^{\prime}\right|}^{*}(\theta) \quad \text { for } \text { a.e. } \quad \theta \in \Theta
$$

If not, we have

$$
\lambda_{j,|k|}^{*}(\theta) \equiv \lambda_{j^{\prime},\left|k^{\prime}\right|}^{*}(\theta) \text { on } \Theta,
$$

since $\lambda_{j,|k|}^{*}(\theta)$ and $\lambda_{j^{\prime},\left|k^{\prime}\right|}^{*}(\theta)$ are analytic on $\Theta$. This implies $S_{j,|k|}^{*}$ $=S_{j^{\prime},\left|k^{\prime}\right|}^{*}$, so $S_{j,|k|}=S_{j^{\prime},\left|k^{\prime}\right|}$ by duality between $S_{j,|k|}$ and $S_{j,|k|}^{*}$. This contradicts Theorem 4.1 which will be given in the Appendix. Thus we have (3.27). Then, by (3.21) of Lemma 3.2,

$$
\lim _{t \rightarrow \infty} \int_{0}^{\infty}\left|G_{j, k}\left(\lambda_{j,|k|}^{*}(\theta) r-t, \theta\right) \cdot G_{j^{\prime}, k^{\prime}}\left(\lambda_{j^{\prime},\left|k^{\prime}\right|}^{*}(\theta) r-t, \theta\right)\right| d r=0
$$


for a.e. $\theta \in \Theta$. On the other hand, there exists a constant $\alpha$ such that

$$
\begin{aligned}
& \left(\lambda_{j,|k|}^{*}(\theta) \lambda_{j^{\prime},\left|k^{\prime}\right|}^{*}(\theta)\right)^{\frac{1}{2}} \\
& \quad \times \int_{0}^{\infty}\left|G_{j, k}\left(\lambda_{j,|k|}^{*}(\theta) r-t, \theta\right) \cdot G_{j^{\prime}, k^{\prime}}\left(\lambda_{j^{\prime},\left|k^{\prime}\right|}^{*}(\theta) r-t, \theta\right)\right| d r \\
& \quad \leqq \\
& \quad \alpha\left\|G_{j, k}(\cdot, \theta)\right\|_{L^{2}(\boldsymbol{R})}\left\|G_{j^{\prime}, k^{\prime}}(\cdot, \theta)\right\|_{L^{2}(\boldsymbol{R})},
\end{aligned}
$$

and

$$
\left\|G_{j, k}(\cdot, \theta)\right\|_{L^{2}(\boldsymbol{R})}, \quad\left\|G_{j^{\prime}, k^{\prime}}(\cdot, \theta)\right\|_{L^{2}(\boldsymbol{R})} \in L^{2}(\Theta) .
$$

Consequently the integral (3.26) converges to zero as $t \rightarrow \infty$ by Lebesgue's Theorem of dominated convergence. Thus we get (3.24). (3.25) can also be verified in a quite similar way. Letting $t \rightarrow \infty$ in (3.23) and applying Proposition 2.2 and (2.24) of Proposition 2.3, we get

$$
\begin{aligned}
\lim _{t \rightarrow \infty} & \mathscr{E}\left(V_{0}+V^{\infty}, C, t\right) \\
& =\lim _{t \rightarrow \infty} \mathscr{E}\left(V_{0}, C, t\right)+\sum_{j=1}^{M} \sum_{|k|=1}^{m_{j}}\left\|\hat{h}_{j, k}\right\|_{L^{2}\left((\operatorname{sgn} k) C_{s_{j,|k|}}\right)} .
\end{aligned}
$$

Then we find by Theorem 1.1

$$
\begin{aligned}
& \mathscr{E}^{\infty}(V, C)=\mathscr{E}\left(V_{0}, C, 0\right) \\
& +\sum_{j=1}^{M} \sum_{|k|=1}^{m_{j}}\left\|\hat{h}_{j, k}\right\|_{L^{2}\left((\operatorname{sgn} k) c_{s_{j,|k|}}\right)}
\end{aligned}
$$

Q.E.D.

Proof of Corollary 1.4. If we introduce new functions

$$
V^{\prime}(t, x)=V(t, x+\bar{x}) \quad \text { and } \quad h^{\prime}(x)=h(x+\bar{x}),
$$

we have

$$
\left\{\begin{array}{l}
\left(I \frac{\partial}{\partial t}+\sum_{j=1}^{n} A_{j} \frac{\partial}{\partial x_{j}}\right) V^{\prime}(t, x)=0 \\
V^{\prime}(0, x)=h^{\prime}(x) \in L^{2}\left(\boldsymbol{R}^{n}\right)
\end{array}\right.
$$

Applying (3.30) to $V^{\prime}$, we find 


$$
\begin{aligned}
\lim _{t \rightarrow \infty} & \mathscr{E}(V, C+\bar{x}, t)=\lim _{t \rightarrow \infty} \mathscr{E}\left(V^{\prime}, C, t\right) \equiv \mathscr{E}^{\infty}\left(V^{\prime}, C\right) \\
& =\mathscr{E}\left(V_{0}^{\prime}, C, 0\right)+\sum_{j=1}^{M} \sum_{|k|=1}^{m_{j}}\left\|\hat{h}_{j, k}^{\prime}\right\|_{L^{2}\left((\operatorname{sgn} k) C_{s_{j,|k|}}\right)}^{2}
\end{aligned}
$$

Now,

$$
\hat{h}^{\prime}(\xi)=\hat{h}(\xi) \exp \{i \bar{x} \xi\}
$$

Hence,

$$
\hat{V}_{0}^{\prime}(\xi)=E_{0}(\xi) \hat{h}^{\prime}(\xi)=\hat{V}_{0}(\xi) \exp \{i \bar{x} \xi\}
$$

This implies that $V_{0}^{\prime}(x)=V_{0}(x+\bar{x})$, and therefore

$$
\mathscr{E}\left(V_{0}^{\prime}, C, 0\right)=\mathscr{E}\left(V_{0}, C+\bar{x}, 0\right) .
$$

Moreover, from the relation

$$
\hat{h}_{j, k}^{\prime}(\xi)=E_{j, k}(\xi) \hat{h}^{\prime}(\xi)=\hat{h}_{j, k}(\xi) \exp \{i \bar{x} \xi\}
$$

we have

$$
\left\|\hat{h}_{j, k}^{\prime}\right\|_{L^{2}\left((\operatorname{sgn} k) C_{\left.S_{j,|k|}\right)}^{2}\right.}=\left\|h_{j, k}\right\|_{L^{2}\left((\operatorname{sgn} k) C_{\left.s_{j,|k|}\right)}^{2}\right.} \cdot
$$

Substituting (3.36) and (3.38) in (3.33), we get

$$
\begin{array}{r}
\lim _{t \rightarrow \infty} \mathscr{E}(V, C+\bar{x}, t)=\mathscr{E}\left(V_{0}, C+\bar{x}, 0\right) \\
+\sum_{j=1}^{M} \sum_{|k|=1}^{m_{j}}\left\|\hat{h}_{j, k}\right\|_{L^{2}\left((\operatorname{sgn} k) C_{S_{j,|k|}}\right)} .
\end{array}
$$

From (3.30) and (3.39) we find (1.20).

Proof of Corollary 1.5. From (1.22) and (3.9), it follows that $V_{0}=0$. From (1.21), (1.22), (3.7) and (3.30), we have

$$
\begin{aligned}
\mathscr{E}\left(V, \boldsymbol{R}^{n}, 0\right)=\|h\|^{2}=\|\hat{h}\|^{2} \\
=\sum_{j=1}^{M} \sum_{|k|=1}^{m_{j}} \int_{\boldsymbol{R}^{n}}\left|E_{j, k}(\xi) \hat{h}(\xi)\right|^{2} d \xi \\
=\sum_{j=1}^{M} \sum_{|k|=1}^{m_{j}} \int_{(\operatorname{sgn} k) C_{s_{j,|k|}}}\left|E_{j, k}(\xi) \hat{h}(\xi)\right|^{2} d \xi
\end{aligned}
$$




$$
=\mathscr{E}^{\infty}(V, C)
$$

Combining this and (1.20), we find

$$
\mathscr{E}^{\infty}(V, C+\bar{x})=\mathscr{E}^{\infty}(V, C)=\mathscr{E}\left(V, \boldsymbol{R}^{n}, 0\right)
$$

Consider the case where the $A_{j}$ are real symmetric. For each $(j, k)$ with $1 \leqq j \leqq M$ and $1 \leqq k \leqq m_{j}$, choose a $\boldsymbol{C}^{N}$-valued function $g_{j, k} \in L^{2}\left(\boldsymbol{R}^{n}\right)$ such that $\operatorname{supp} \hat{g}_{j, k}(\xi) \subset C_{S_{j, k}}$ and define

$$
g_{j,-k}(x)=\overline{g_{j, k}(x)}
$$

Then we have

$$
\hat{g}_{j,-k}(\xi)=\overline{\hat{g}_{j, k}(-\xi)}
$$

Put

$$
\hat{h}(\xi)=\sum_{j=1}^{M} \sum_{|k|=1}^{m_{j}} E_{j, k}(\xi) \hat{g}_{j, k}(\xi)
$$

Then the function $h \in L^{2}\left(\boldsymbol{R}^{n}\right)$ satisfies (1.22). Moreover we have from (3.13)

$$
\begin{aligned}
\overline{\hat{h}(-\xi)} & =\sum_{j=1}^{M} \sum_{|k|=1}^{m_{j}} \overline{E_{j, k}(-\xi) \hat{g}_{j, k}(-\xi)} \\
& =\sum_{j=1}^{M} \sum_{|k|=1}^{m_{j}} E_{j,-k}(\xi) \hat{g}_{j,-k}(\xi) \\
& =\hat{h}(\xi) .
\end{aligned}
$$

this implies that $h(x)$ is a real valued function.

Q.E.D.

\section{§4. Appendix}

Theorem 4.1. Let $P(\lambda, \xi)$ be a polynomial of $(n+1)$-variables $\left(\lambda, \xi_{1}, \ldots, \xi_{n}\right)$ with complex coefficients and let

$$
P(\lambda, \xi)=\prod_{k=0}^{p}\left(Q_{k}(\lambda, \xi)\right)^{\alpha_{k}}
$$

be the factorization into irreducible polynomials in the polynomial ring 
$\boldsymbol{C}[\lambda, \xi]$ of $(n+1)$-variables over the complex number field. Let $\Omega$ be a domain in $\boldsymbol{R}^{n}$ and assume that the factorization into linear factors in $\lambda$ of $P(\lambda, \xi)$ admits the form

$$
P(\lambda, \xi)=\prod_{j=0}^{q}\left(\lambda-\lambda_{j}(\xi)\right)^{\beta_{j}} \quad \text { for all } \xi \in \Omega,
$$

where the $\beta_{j}$ are constant positive integers and the $\lambda_{j}(\xi)$ are analytic functions on $\Omega$ such that

$$
i \neq j \Longrightarrow \lambda_{i}(\xi) \not \equiv \lambda_{j}(\xi)
$$

Then we have

$$
\prod_{k=0}^{p} Q_{k}(\lambda, \xi)=\prod_{j=0}^{q}\left(\lambda-\lambda_{j}(\xi)\right) \quad \text { in } \quad \Omega
$$

For the proof we need the following

Lemma 4.2 (Hörmander [1] Appendix). If a polynomial $Q(\lambda, \xi)$ has no multiple factor, there exist a polynomial $R(\xi)$, not identically zero, such that the zeros of $Q(\lambda, \xi)$ as a polynomial in $\lambda$ are all different for every $\xi$ with $R(\xi) \neq 0$.

\section{Proof of Theorem 4.1. Set}

$$
Q(\lambda, \xi)=\prod_{k=0}^{p} Q_{k}(\lambda, \xi)
$$

Then there exists, by the above lemma, a polynomial $R(\xi)$ such that the roots of the equation $Q(\lambda, \xi)=0$ in $\lambda$ are all different for $\xi$ with $R(\xi) \neq 0$. Put

$$
\Omega_{0}=\{\xi \in \Omega ; R(\xi)=0\}
$$

and

$$
\Omega_{1}=\bigcup_{i<j}\left\{\xi \in \Omega ; \lambda_{i}(\xi)=\lambda_{j}(\xi)\right\}
$$

Then, $\Omega_{0}$ and $\Omega_{1}$ are closed in $\Omega$ and have no interior points, so the set $\Omega \backslash\left(\Omega_{0} \cup \Omega_{1}\right)$ is a non empty open set and we have 


$$
\prod_{j=0}^{q}\left(\lambda-\lambda_{j}(\xi)\right)=Q(\lambda, \xi) \quad \text { for } \quad \xi \in \Omega \backslash\left(\Omega_{0} \cup \Omega_{1}\right)
$$

Since the $\lambda_{j}(\xi)$ and $Q(\lambda, \xi)$ are analytic functions of $\xi$, we have (4.4).

Q.E.D.

\section{References}

[1] Hörmander, L., Linear Partial Differential Operators, Berlin-Göttingen-Heidelberg: Springer 1964.

[2] Littman, W., Fourier transforms of surface-carried measures and differentiability of surface averages, Bull. Amer. Math. Soc., 69 (1963), 766-770.

[3] Matsumura, M., On some properties of the fundamental solutions for stationary and non-stationary wave propagation problems. I. La Funkcialaj Ekvacioj, 24 (1972), 433-524 (in Japanese).

[4] —- Asymptotic behavior at infinity for Green's functions of first order systems with characteristics of nonuniform multiplicity, Publ. RIMS, Kyoto Univ., $\mathbb{1 2}$ (1976), 317-377.

[5] Matsuura, S., On non-strict hyperbolicity, Conf. on Functional Analysis and Related Topics, Tokyo 1969, Math. Soc. Japan (1970), 171-176.

[6] Sternberg, S., Lectures on Differential Geometry, Englewood Cliffs, N. J.: Prentice-Hall 1964.

[7] Wilcox, C. H., Wave operators and asymptotic solutions of wave propagation problems of classical physics, Arch. Rational Mech. Anal., 22 (1966), 37-78.

[8] — Asymptotic wave functions and energy distributions for the d'Alembert wave equation, ONR Technical Summary Rept. \$24, Univ. of Utah (March 1974).

[9] — Scattering Theory for the d'Alembert Equation in Exterior Domains, Lecture Notes in Mathematics, Vol. 442, Berlin-Heidelberg-New York: Springer 1975. 
